\title{
Rno-microRNA-30c-5p promotes myocardial ischemia reperfusion injury in rats through activating NF-kB pathway and targeting SIRT1
}

Jianfeng Chen ${ }^{1}$, Mingming Zhang ${ }^{1}$, Shouyan Zhang ${ }^{1 *} \mathbb{D}$, Junlong $\mathrm{Wu}^{2}$ and Shufeng Xue

\begin{abstract}
Background: This study aimed to investigate the regulatory effect of rno-microRNA-30c-5p (rno-miR-30c-5p) on myocardial ischemia reperfusion (IR) injury in rats and the underlying molecular mechanisms.

Methods: A rat model of myocardial IR injury was established. The infarct size was detected by 2,3,5triphenyltetrazolium chloride staining. The pathologic changes of myocardial tissues were detected by hematoxylineosin staining. The apoptosis of myocardial cells was measured by TUNEL staining and flow cytometry. The mRNA expression of rno-miR-30c-5p and Sirtuin 1 (SIRT1) was detected by quantitative real-time PCR. The levels of IL-1 $\beta$, IL-6 and TNF-a were detected by enzyme linked immunosorbent assay. The protein expression of Bax, BCl-2, caspase-3, p-IKBa, IkBa, p-NF-kB p65, NF-kB p65 and SIRT1 was detected by Western blot. The interaction between rno-miR-30c-5p and SIRT1 was predicted by TargetScan, and further identified by dual luciferase reporter gene and RNA immunoprecipitation assay.
\end{abstract}

Results: The myocardial IR injury model was successfully established in rats. IR induced the myocardial injury in rats and increased the expression of rno-miR-30c-5p. Overexpression of rno-miR-30c-5p enhanced the inflammation, promoted the apoptosis, and activated NF-KB pathway in IR myocardial cells. SIRT1 was the target gene of rno-miR30c-5p. Silencing of SIRT1 reversed the effects of rno-miR-30c-5p inhibitor on the apoptosis and NF-kB pathway in IR myocardial cells.

Conclusions: Rno-miR-30c-5p promoted the myocardial IR injury in rats through activating NF-KB pathway and down-regulating SIRT1.

Keywords: Myocardial ischemia reperfusion injury, Rno-miR-30c-5p, Inflammation, Apoptosis, SIRT1, NF-kB pathway

\footnotetext{
*Correspondence: zgangshouyan289@163.com

'Department of Cardiology, Luoyang Central Hospital Affiliated to

Zhengzhou University, No. 288, Zhongzhou Middle Road, Luoyang City

471000, Henan Province, China

Full list of author information is available at the end of the article
}

(c) The Author(s). 2020 Open Access This article is licensed under a Creative Commons Attribution 4.0 International License, which permits use, sharing, adaptation, distribution and reproduction in any medium or format, as long as you give appropriate credit to the original author(s) and the source, provide a link to the Creative Commons licence, and indicate if changes were made. The images or other third party material in this article are included in the article's Creative Commons licence, unless indicated otherwise in a credit line to the material. If material is not included in the article's Creative Commons licence and your intended use is not permitted by statutory regulation or exceeds the permitted use, you will need to obtain permission directly from the copyright holder. To view a copy of this licence, visit http://creativecommons.org/licenses/by/4.0/. The Creative Commons Public Domain Dedication waiver (http://creativecommons.org/publicdomain/zero/1.0/) applies to the data made available in this article, unless otherwise stated in a credit line to the data. 


\section{Background}

Ischemic heart disease is a series of diseases characterized by myocardial ischemia, such as angina pectoris and myocardial infarction [1]. Recently, reperfusion of the ischemic myocardium is one of the most common therapeutic strategies for ischemic heart diseases [2]. Although restoring blood flow in time can relieve myocardial infarction to a great extent, the prognosis of patients remains poor due to the ischemia reperfusion (IR) injury on myocardium [3]. Therefore, it is urgent to find out novel therapeutic methods and targets for myocardial IR injury.

The genome-wide investigations of genetic variants, epigenetic modifications, and gene expression profiles optimize the search for novel diagnostic or therapeutic targets for IR injury in the post-genomic era [4]. MicroRNAs (miRNAs) are a class of small endogenous noncoding RNAs with 19-25 nucleotides in length, which modulate gene expression at the post-transcriptional level $[5,6]$. A systematic comparison of IR injuryinduced miRNA expression changes in rats identifies several potential cardioprotective miRNA targets (protectomiRs), including Rno-miR-125b*, - 139-3p, - 320, -532-3p, and-188 [7]. By using bioinformatics methods based on topological or network dynamical approaches, the mRNA targets of protectomiRs can be predicated. Nevertheless, all unbiased omics approaches and their bioinformatic evaluation need to be verified by rigorous experimental validation at the transcript and protein levels [8].

Recently, studies have indicated that miRNAs play important regulatory roles in myocardial IR injury [9]. Yuan et al. [10] have proved that the inhibition of rnomiR-181b-5p protects cardiomyocytes against I/R injury through targeting AKT3 and PI3KR3. Zhao et al. [11] have reported that $\mathrm{mmu}-\mathrm{miR}-374 \mathrm{a}$ protects against myocardial IR injury in mice via targeting MAPK6 pathway. Song et al. [12] have indicated that rno-miR-30b overexpression has anti-apoptotic effect on cardiomyocytes at early phase of myocardial IR injury in a rat model. MiR-30c-5p is another subtype of miR-30 that also involved in the process of IR injury. Zhou et al. [13] have proved that rno-miR-30c-5p is a potential diagnostic marker for I/R-induced kidney injury in rats. Li et al. [14] have shown that hydrogen sulfide protects spinal cord and induces autophagy in a rat model of spinal cord IR injury via regulating rno-miR-30c-5p. However, the regulatory effect and mechanism of rno-miR-30c-5p on myocardial IR injury remain unclear.

Nuclear factor $\kappa \mathrm{B}(\mathrm{NF}-\mathrm{\kappa} \mathrm{B})$ is involved in the regulation of multiple biological functions including innate immunity, inflammation, cell proliferation and apoptosis [15, 16]. Accumulating researches have revealed that myocardial IR injury is associated with the activation of NF- $\mathrm{BB}$
[17]. In addition, emerging evidence has indicated that miRNAs play vital roles in myocardial IR injury by regulating $\mathrm{NF}-\mathrm{\kappa} \mathrm{B}$ pathway. For instance, mmu-miR-146a overexpression reduces myocardial IR injury via inhibiting the activation of NF-kB pathway [18]. However, whether the regulatory effect of rno-miR-30c-5p on myocardial IR injury is involved in NF- $\mathrm{kB}$ pathway is unknown.

In this study, we explored the regulatory effect of rnomiR-30c-5p on myocardial IR injury in rats, as well as the underlying molecular mechanisms. Our results indicated that rno-miR-30c-5p promoted the myocardial IR injury in rats through activating $N F-\kappa B$ pathway and down-regulating SIRT1. Our findings may provide a new theoretical foundation for the treatment of myocardial IR injury in clinical practice.

\section{Methods}

Animals

Male Sprague-Dawley (SD) rats (weighting 180-200 g) were provided by Peking University Laboratory Animal Center. All rats were kept at $22-24{ }^{\circ} \mathrm{C}$ and $55-60 \%$ humidity on a $12 \mathrm{~h}$ light-dark cycle with free access to water and food. At the end of the study, all rats (220$270 \mathrm{~g}$ ) were anesthetized by an intraperitoneal injection of $50 \mathrm{mg} / \mathrm{kg}$ pentobarbital sodium, and then sacrificed by cervical dislocation. All animal experiments were conducted strictly in accordance with the National Institutes of Health guide for the care and use of Laboratory animals.

\section{Establishment of the myocardial IR model in rats}

Rats weighing 200-240 g were used to establish the IR model. Briefly, rats were anesthetized with pentobarbital sodium (50 mg/kg, i.p.). The left anterior descending coronary artery (LAD) was ligated using 6-0 silk suture slipknot for $30 \mathrm{~min}$, and then reperfused for $2 \mathrm{~h}$. Myocardial ischemia was confirmed by the appearance of regional epicardial cyanosis over the myocardial surface and by arrhythmia. Successful reperfusion was confirmed by the disappearance of epicardial cyanosis and the production of epicardial hyperemia and arrhythmia (IR group). Rats undergoing thoracotomy without LAD ligation were considered as the Sham group.

\section{Hemodynamic examination}

One week after modeling, the hemodynamic parameters including left ventricular ejection fraction (LVEF), left ventricular systolic pressure (LVSP), left ventricular enddiastolic volume (LVEDV), left ventricular end-systolic volume (LVESV), left ventricular end-diastolic pressure (LVEDP), the maximum up rate of left ventricular pressure $\left(+\mathrm{dP} / \mathrm{dt}_{\max }\right)$, and the maximum down rate of left 
ventricular pressure $\left(-\mathrm{dP} / \mathrm{dt}_{\max }\right)$ were measured using a Vevo770 scanner (VisualSonics, Toronto, Canada).

\section{Infarct size measurement}

The infarct size was detected using 2,3,5-triphenyltetrazolium chloride (TTC) (Sangon, Shanghai, China) staining. Briefly, the ventricle was sliced into pieces with equal thickness. The slices were then incubated in $2 \%$ TTC for $15 \mathrm{~min}$ in the dark and fixed in 10\% formaldehyde for $10 \mathrm{~min}$. The infarct area was measured by an image analyzer. The infarct size was calculated as the ratio of the infarct area and total area (\%).

\section{Hematoxylin-eosin (HE) staining}

The ventricle was fixed in $4 \%$ formaldehyde overnight at $4{ }^{\circ} \mathrm{C}$. Followed by dehydration, vitrification, and paraffinembedding, the tissue samples were cut into $5 \mu \mathrm{m}$-thick slices. The sections were then deparaffined in xylene, rehydrated in gradient ethanol, and stained with hematoxylin for $4 \mathrm{~min}$ and Eosin for $2 \mathrm{~min}$. The histopathological changes were observed under a light microscope $(400 \times)$.

\section{Terminal dexynucleotidyl transferase-mediated dUTP nick end labeling (TUNEL) staining}

Cell apoptosis was detected using a TUNEL kit (Beyotime, Shanghai, China). Briefly, the paraffin-embedded tissue sections were deparaffined in xylene, and rehydrated in gradient ethanol. The sections were then incubated with DNase-free Proteinase K for 20 min, with 3\% hydrogen peroxide (in PBS) for $10 \mathrm{~min}$, and with TUNEL mix for $60 \mathrm{~min}$. After $30 \mathrm{~min}$ of incubation with Streptavidin-HRP, the apoptotic cells were visualized using diaminobenzidine, and re-stained with hematoxylin. The apoptotic cells were counted under a light microscope $(400 \times)$ at five randomly selected fields.

\section{Isolation of IR myocardial cells}

The myocardial tissues at the ischemic site were collected and homogenated. The tissue homogenate was digested with collagenase IV $(0.45 \mathrm{mg} / \mathrm{ml})$ containing $0.1 \%$ trypsin and $15 \mu \mathrm{g} / \mathrm{ml}$ DNase I. After centrifugation, the residue (myocardial cells) was collected. Myocardial cells were cultured in RPMI 1640 medium (Gibco, USA) containing $15 \%$ FBS, and maintained in an incubator at $37^{\circ} \mathrm{C}$ with $5 \% \mathrm{CO}_{2}$.

\section{Cell transfection and grouping}

The rno-miR-30c-5p mimics, rno-miR-30c-5p inhibitor, SIRT1 siRNA1-3 and the negative controls (mimics NC, inhibitor NC and si-NC) were purchased from Genepharma (Shanghai, China). IR myocardial cells were seeded into 24 -well plates $\left(1 \times 10^{5} /\right.$ well $)$, and cultured until $80 \%$ confluence. Cells were then transfected with the above agents using Lipofectamine 3000. IR myocardial cells were randomly divided into 9 groups: IR (no treatment), inhibitor NC, rno-miR-30c-5p inhibitor, mimics NC, rno-miR-30c-5p mimics, si-NC + inhibitor $\mathrm{NC}$, siRNA2 + inhibitor NC, siRNA2 + rno-miR-30c-5p inhibitor, and si-NC + rno-miR-30c-5p inhibitor group. After $48 \mathrm{~h}$ of transfection, cells were used for subsequent experiments.

\section{Flow cytometry}

Myocardial cells were washed with PBS twice and then stained with Annexin V-fluorescein isothiocyanate (FITC) and propidium iodide (PI) for $15 \mathrm{~min}$ in the dark. The apoptosis was detected by a flow cytometer (Beckman Coulter, USA).

\section{Enzyme linked immunosorbent assay (ELISA)}

The myocardial cells and tissues were homogenated and maintained on ice. The levels of inflammatory factors including TNF- $\alpha$, IL- $1 \beta$ and IL- 6 were detected using specific ELISA kits (Thermo Fisher Scientific, USA) in accordance with the manufacturer's instructions.

\section{Quantitative real-time PCR}

Total RNA was extracted from myocardial cells and tissues using TRIZOL (Invitrogen, USA). Total RNA was then reverse-transcribed into cDNA using a Reverse Transcription Kit (Thermo Fisher Scientific, USA). qRTPCR was performed on a PCR instrument (Bio-Rad, USA) using SYBR Green Mixture (Roche, Switzerland). Primers were shown as follows: rno-miR-30c-5p F: $5^{\prime}$ GGGGTGTAAACATCCTACAC-3'， R: 5'-GTGGAG TCGGCAATTGCACT-3'; U6 F: 5'-GCTTCGGCAG CACATATACTAAAAT-3', R: 5'-CGCTTCAC GAATTTG CGTGTCAT-3'; SIRT1 F: 5'-AAGGAG CAGATTAGTAAGC-3', R: 5'-TAGAGGATAAGGCG TCAT-3'; GAPDH F: 5'-GACGGCCGCATCTT CTTGT-3', R: 5'-CACACCGACCTTCACCATTTT-3'. GAPDH and U6 with stable expression were used as internal controls of SIRT1 and rno-miR-30c-5p, respectively.

\section{Western blot}

Total protein was extracted from myocardial cells and tissues using RIPA lysis buffer (Beyotime, Shanghai, China). The protein samples $(50 \mu \mathrm{g})$ were separated by $10 \%$ SDS-PAGE and then transferred onto polyvinylidenedifluoride membrane. After blocked with 5\% skim milk in TBST for $2 \mathrm{~h}$, the membrane was incubated with specific primary antibody (anti-Bax, 1:1000, 14,796; antiBcl-2, 1:1000, 4228 s; anti-ІкB $\alpha, 1: 500$, \#4814; anti-pIкB $\alpha, 1: 500, \# 2859$; anti-SIRT1, 1:1000, \#2310, Cell signal, USA; anti-NF- $\mathrm{kB}$ p65, 1:1000, SAB4502610; antip-NF-кB p65, 1:1000, SAB4301496, Sigma Aldrich, USA; 
anti-caspase-3, 1:1000, sc-271,759; anti- $\beta$-actin, 1:1000, sc-517,582, Santa Cruz, USA) overnight at $4{ }^{\circ} \mathrm{C}$. After washed with TBST for three times, the membrane was incubated with horseradish peroxidase (HRP)-labeled secondary antibody for $2 \mathrm{~h}$ at $25^{\circ} \mathrm{C}$. The protein bands were visualized using a HRP kit and quantified by an ECL system (Thermo Fisher Scientific, USA).

\section{TargetScan prediction}

The targets of rno-miR-30c-5p were predicted using TargetScan 7.1 (http://www.targetscan.org/vert_71/). A total of 1249 transcripts containing 1835 sites were predicted (Table S1). A target gene SIRT1 (ENST00000212015.6) was selected due to its important role in myocardial IR injury (Table S2).

\section{Dual luciferase reporter gene (DLR) assay}

DLR assay was used to identify the targeting relationship between SIRT1 and rno-miR-30c-5p. The fragment of SIRT1, containing the binding site was amplified and cloned into pmirGLO luciferase vector (Promega, USA) to construct wild pmirGLO-WT-SIRT1-3'-UTR (SIRT1-Wt) and mutant pmirGLO-MUT-SIRT1-3'-UTR (SIRT1-Mt). Myocardial cells were co-transfected with SIRT1-Wt/Mt and rno-miR-30c-5p mimics/mimics NC using Lipofectamine 3000. Myocardial cells were randomly divided into 4 groups: SIRT1-Mt + rno-miR-30c-5p mimics, SIRT1-Mt + mimics NC, SIRT1-Wt + rno-miR-30c-5p mimics, and SIRT1-Wt + mimics NC group. After $48 \mathrm{~h}$ of transfection, the luciferase activity was measured using a dual luciferase kit (Promega).

\section{RNA immunoprecipitation (RIP) assay}

RIP assay was performed using a Magna RIP Kit (Millipore, USA). Briefly, myocardial cells were lysed in lysis buffer. The cell lysate was then incubated with antiAgo 2 or IgG-coated beads at $4{ }^{\circ} \mathrm{C}$ for $2 \mathrm{~h}$. After washed with PBS, the RNA-protein-beads complexes were isolated using Trizol. The expression of rno-miR-30c-5p and SIRT1 was measured by qRT-PCR.

\section{Statistical analysis}

Three independent repetitions were conducted for each sample. Data were expressed as mean \pm standard deviation (SD), and analyzed using SPSS 22.0 Statistical Software (Chicago, IL). Differences among multi-groups were analyzed by one-way ANOVA followed by Tukey's post hoc test. Differences between two groups were analyzed by Student's $t$ test. The level of statistical significance was set at $p<0.05$.

\section{Results}

IR induces myocardial injury in rats

As shown in Fig. 1a, the levels of LVEF, LVSP, $+\mathrm{dP} /$ $\mathrm{dt}_{\max }$ and $-\mathrm{dP} / \mathrm{dt}_{\max }$ were significantly lower, and the levels of LVEDV, LVESV and LVEDP were significantly higher in the IR group than those in the Sham group $(P<0.05)$. The infarct size was significantly higher in the IR group than that in the Sham group $(P<0.05)$ (Fig. $1 b)$. HE staining showed that the myocardial fibers in the Sham group were orderly arranged without inflammatory cell infiltration. Disorganized myocardial fibers accompanied with obvious inflammatory cell infiltration were observed in the IR group (Fig. 1c). The levels of IL6 , IL-1 $\beta$ and TNF- $\alpha$ in the Sham group were significantly higher than those in the IR group $(P<0.05)$ (Fig. $1 d)$. In addition, TUNEL assay showed that IR significantly promoted the apoptosis of myocardial cells $(P<$ $0.05)$ (Fig. 1e). The protein expression of Bax, caspase-3, and p-NF- $\mathrm{kB}$ p65/NF- $\mathrm{kB}$ p 65 was significantly increased, and the protein expression of $\mathrm{Bcl}-2$ and $\mathrm{p}-\mathrm{I \kappa}_{\kappa} \alpha / \mathrm{I \kappa B \alpha}$ was significantly decreased in the IR group compared with that in the Sham group $(P<0.05)$ (Fig. 1f and h). Note worthily, the expression of rno-miR-30c-5p was significantly higher in the IR group than that in the Sham group $(P<0.05)$ (Fig. $1 \mathrm{~g})$. All these results suggested that IR could induce the myocardial injury in rats.

\section{Rno-miR-30c-5p enhances the inflammation, promotes the apoptosis, and activated NF-KB pathway in IR myocardial cells}

As shown in Fig. 2a, the expression of rno-miR-30c-5p in IR myocardial cells was significantly decreased in the rno-miR-30c-5p inhibitor group, and increased in the rno-miR-30c-5p mimics group compared with the IR group $(P<0.05)$. The expression of rno-miR-30c-5p was not significantly influenced by the transfection of either inhibitor NC or mimics NC (Fig. 2a). The levels of IL-6, IL- $1 \beta$ and TNF- $\alpha$ were significantly decreased in the rno-miR-30c-5p inhibitor group, and were significantly increased in the rno-miR-30c-5p mimics group compared with those in the IR group $(P<0.05)$ (Fig. 2b). The apoptotic index was significantly lower in the ron-miR$30 c-5 p$ inhibitor group and was significantly higher in the rno-miR-30c-5p mimics group than that in the IR group $(P<0.05)$ (Fig. 2c). In addition, the transfection of rno-miR-30c-5p inhibitor significantly decreased the protein expression of Bax, caspase- 3 and $p-N F-k B$ p65/ $\mathrm{NF}-\mathrm{kB}$ p65, and increased the protein expression of Bcl2 and $p-I \kappa B \alpha / I \kappa B \alpha$ in IR myocardial cells. The effect of rno-miR-30c-5p mimics on the expression of the above proteins was opposite to that of rno-miR-30c-5p inhibitor $(P<0.05)$ (Fig. $2 \mathrm{~d}$ and e). These results indicated that rno-miR-30c-5p might enhance the inflammation, 


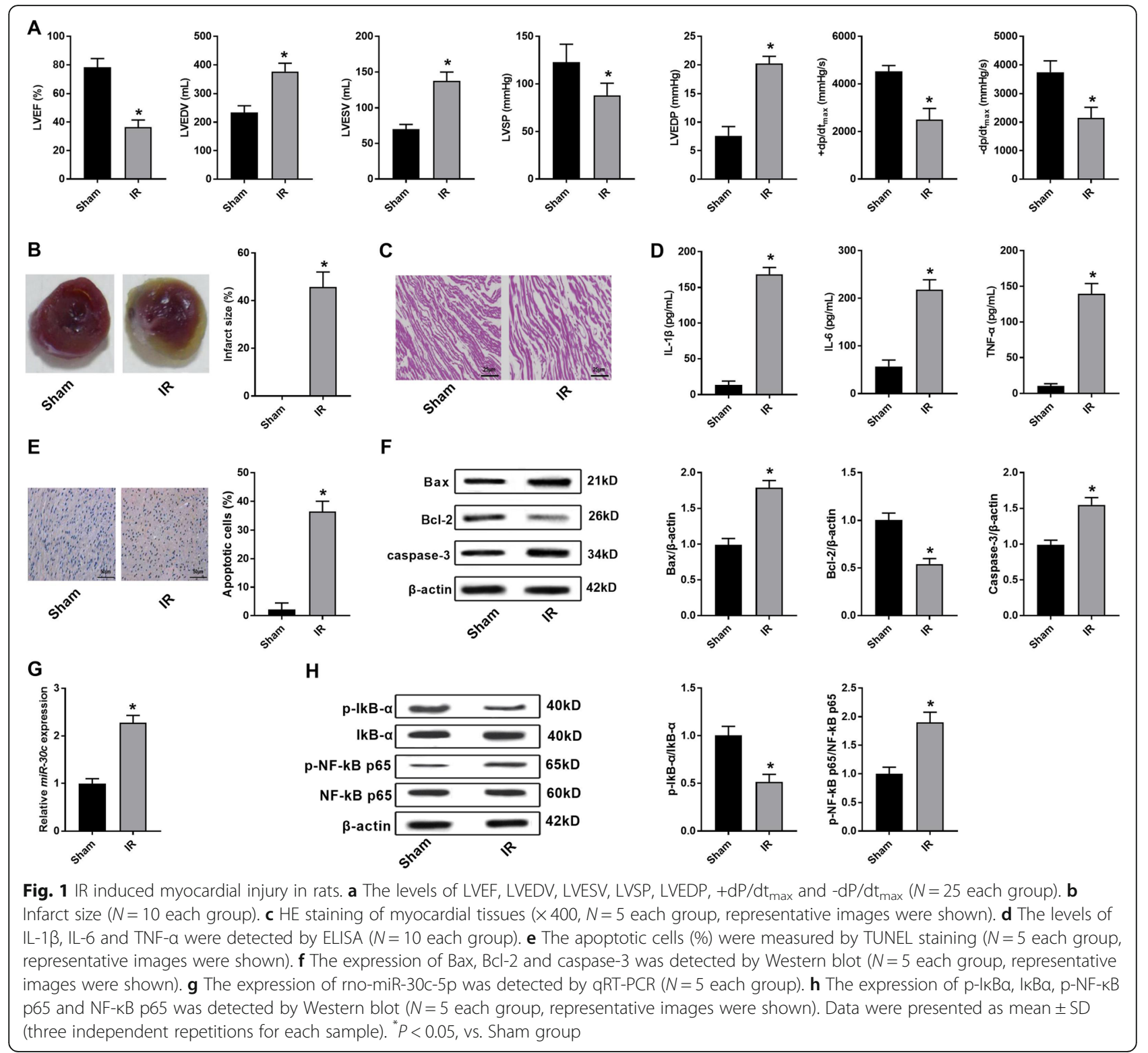

promote the apoptosis and activate NF- $\mathrm{kB}$ pathway in IR myocardial cells.

\section{SIRT1 is the target gene of rno-miR-30c-5p}

As shown in Fig. 3a, the expression of SIRT1 in the IR group was significantly lower than that in the Sham group $(P<0.05)$. The expression of rno-miR-30c-5p was negatively correlated with the expression of SIRT1 $(P<$ 0.05) (Fig. 3b). The transfection of rno-miR-30c-5p inhibitor and rno-miR-30c-5p mimics significantly increased and decreased the expression of SIRT1 in IR myocardial cells at the mRNA and protein level, respectively $(P<0.05)$ (Fig. $3 \mathrm{c})$. A binding site at 3 '-UTR of SIRT1 was predicted on rno-miR-30c-5p by TargetScan (Fig. 3d). DLR assay showed that the luciferase activity was significantly reduced in the SIRT1-Wt + rno-miR30c-5p mimics group compared with that in the SIRT1$\mathrm{Wt}+\mathrm{NC}$-mimics group $(P<0.05)$ (Fig. 3e). RIP assay further indicated the expression of SIRT1 and rno-miR$30 c-5 p$ was significantly decreased in the Anti-IgG group compared with that in the Input group $(P<0.05)$ (Fig. 3e). All these results suggested that SIRT1 was the target gene of rno-miR-30c-5p.

Silencing of SIRT1 reversed the effects of rno-miR-30c-5p inhibitor on the apoptosis and NF-KB pathway in IR myocardial cells

SIRT1 was silenced in IR myocardial cells by the transfection of siRNA1, 2 and 3. As shown in Fig. 4a, the protein expression of SIRT1 was significantly decreased 


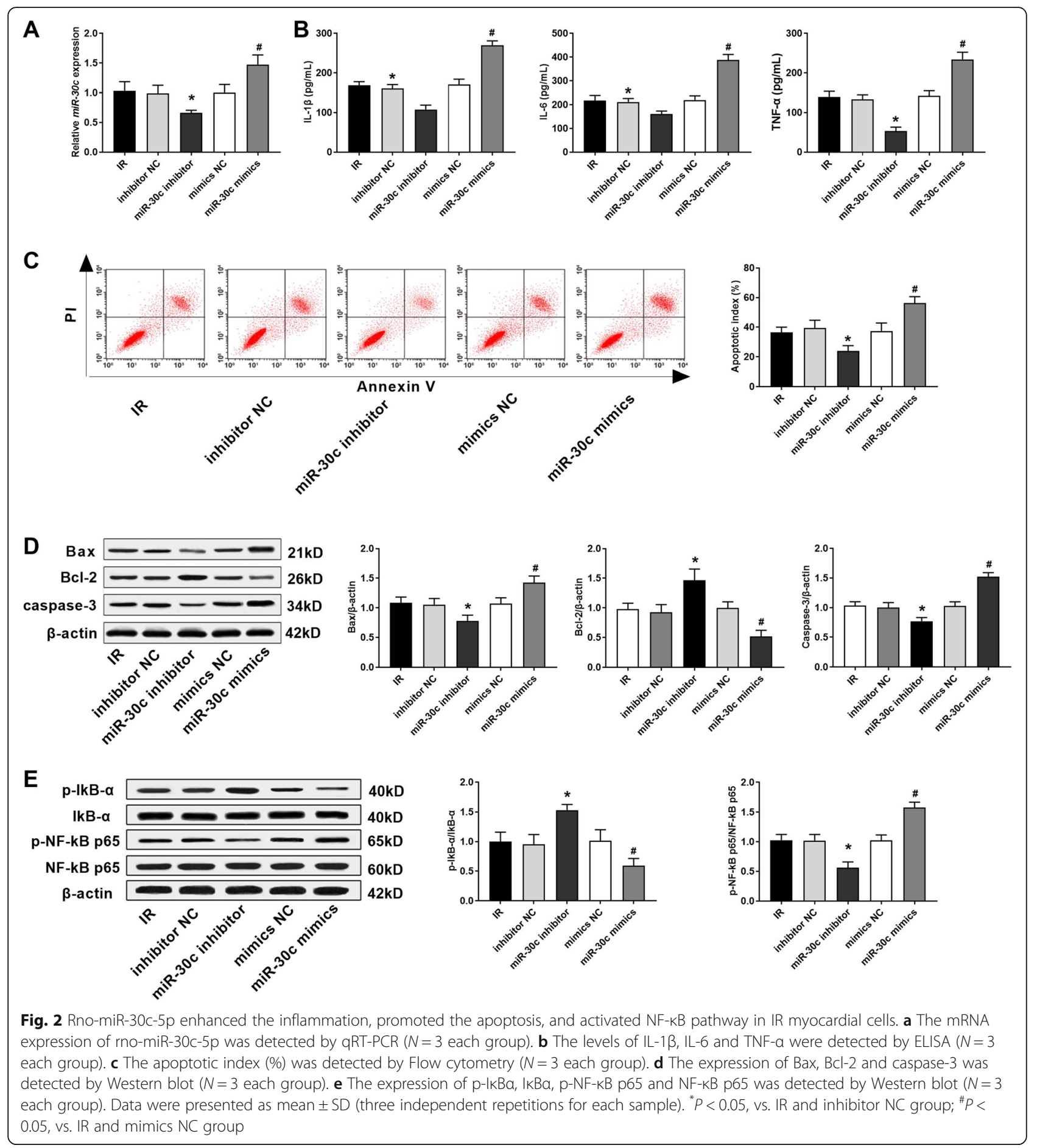

by the transfection of siRNA1, 2 or $3(P<0.05)$. siRNA2 with relatively high silence efficiency was used for subsequent experiments. Compared with the si-NC + inhibitor $\mathrm{NC}$ group, the apoptotic index was significantly increased in the siRNA2 + inhibitor NC group, and was significantly decreased in the si-NC+ rno-miR-30c-5p inhibitor group $(P<0.05)$. In addition, the protein expression of Bax, caspase-3, and p-NF- $\mathrm{kB}$ p65/NF- $\mathrm{kB}$ p65 was significantly increased in the siRNA2 + inhibitor NC group, and was significantly decreased in the si-NC+ rno-miR-30c-5p inhibitor group compared with the si$\mathrm{NC}+$ inhibitor NC group $(P<0.05)$. The protein expression of $\mathrm{Bcl}-2$ and $\mathrm{p}$-IкB $\alpha / \mathrm{I} \kappa \mathrm{B} \alpha$ was opposite to that of Bax in different groups $(P<0.05)$ (Fig. $4 \mathrm{~b}$ and $\mathrm{d})$. Note worthily, the effects of rno-miR-30c-5p inhibitor on the apoptosis and NF-kB pathway were reversed by the 


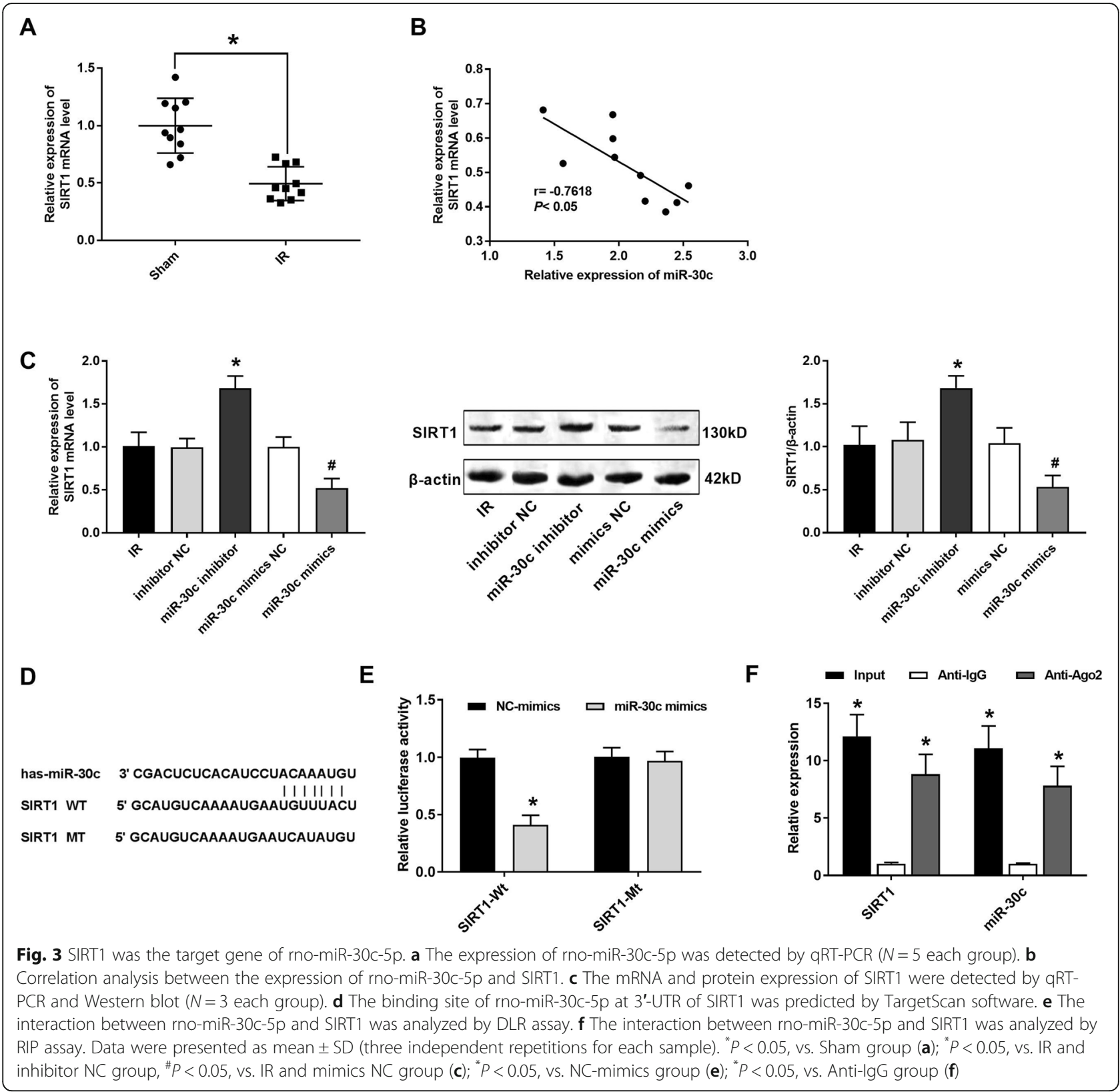

transfection of siRNA2 in IR myocardial cells $(P<0.05)$ (Fig. 4b-d). All these results suggested rno-miR-30c-5p could promote the apoptosis, and activated NF- $\mathrm{kB}$ pathway in IR myocardial cells by targeting SIRT1.

\section{Discussion}

Myocardial infarction is one of the most common causes of death worldwide [19].

The therapeutic outcomes of patients receiving reperfusion are greatly limited by the occurrence of myocardial IR injury. It is urgent to explore the potential molecular mechanisms involving myocardial IR injury, and identify novel therapeutic targets. In this study, we demonstrated that rno-miR-30c-5p could promote the myocardial IR injury in rats through activating NF-kB pathway and down-regulating SIRT1.

Myocardial IR injury often leads to inflammation, and the inflammatory cascade reaction further induces the apoptosis of myocardial cells [20, 21]. MiRNAs exert important roles in myocardial I/R injury through regulating inflammation and cell apoptosis [22, 23]. For example, lentivirus expressing mmu-miR-146a attenuates I/R-induced myocardial apoptosis and inflammatory cytokine production in mice [18]. Intramyocardial injection of mmu-miR322 mimics diminishes cardiac apoptosis and reduces infarct size in IR mice [24]. Overexpression of 


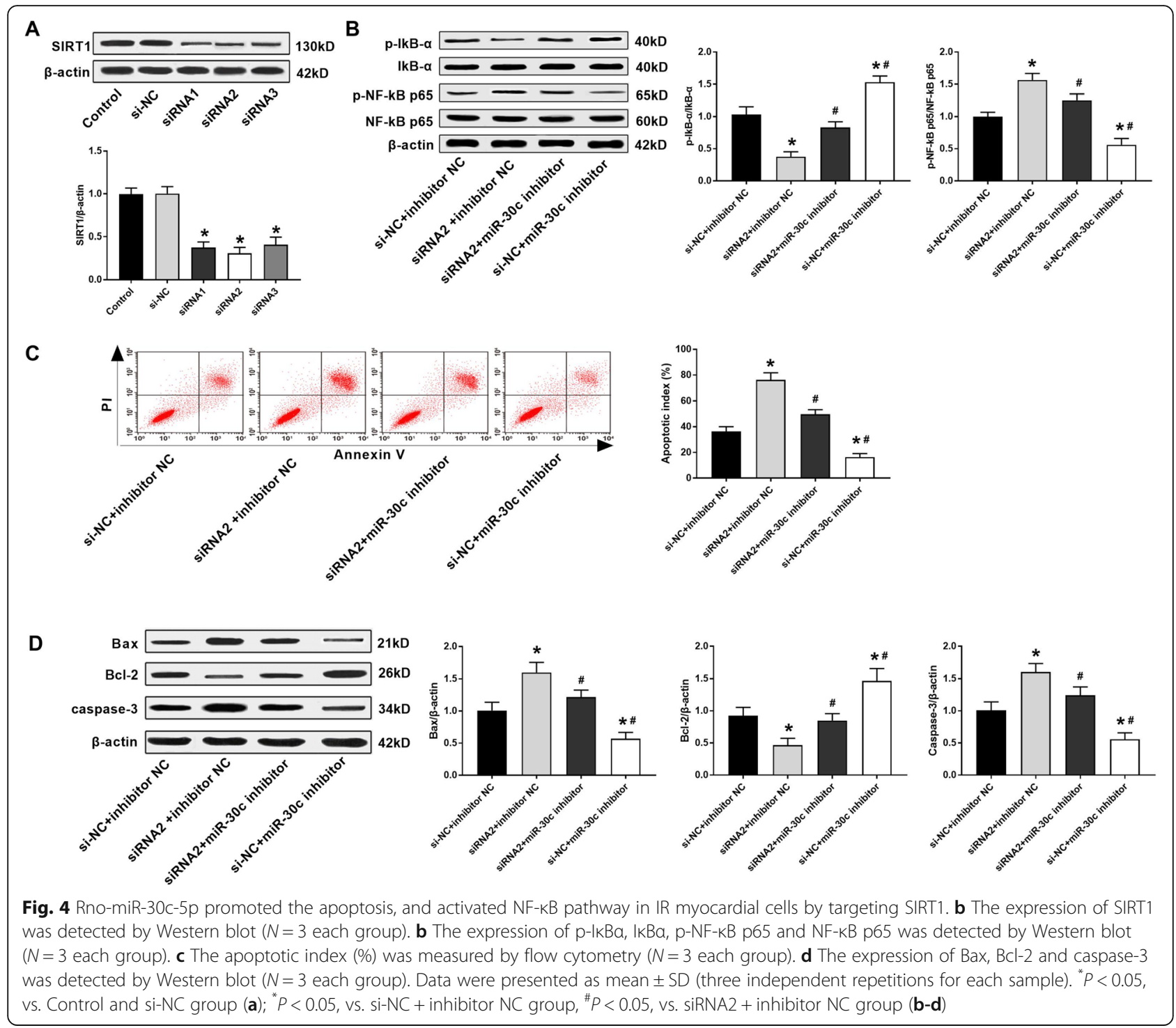

rno-miR-144 significantly reduces the myocardial injury and apoptosis in IR rats [25]. Mmu-miR-24-3p decreases the infarct area and inhibits cell apoptosis in mice with myocardial IR injury. In this study, we found that the expression of rno-miR-30c-5p was significantly upregulated in rats with myocardial IR injury. In vitro experiments confirmed that rno-miR-30c-5p enhances the inflammation and promotes the apoptosis of IR myocardial cells. Our findings indicate that rno-miR$30 c-5 p$ may enhance the myocardial IR injury via promoting inflammation and cell apoptosis. The promoting role of rno-miR-30c-5p on myocardial IR injury is consistent with that on I/R-induced kidney and spinal cord injury. Zhou et al. [13] have proved that ron-miR-30c-5p is up-regulated in rats with $\mathrm{I} / \mathrm{R}$-induced kidney injury. $\mathrm{Li}$ et al. [14] have shown that hydrogen sulfide protects spinal cord and induces autophagy in a rat model of spinal cord IR injury via down-regulating rno-miR-30c5p. Silencing of ron-miR-30c-5p may be a potential therapeutic strategy for myocardial IR injury.

$\mathrm{NF}-\mathrm{KB}$ is involved in the regulation of multiple biological processes including innate immunity, inflammation, cell proliferation and apoptosis $[15,16]$. Under normal physiological condition, inactive NF- $\mathrm{kB}$ complexes are retained in the cytoplasm by binding to inhibitor of $\kappa B$ (ІкB) proteins [26]. The stimuli can promote the phosphorylation and subsequent degradation of $\mathrm{I} \kappa \mathrm{B} \alpha$, and subsequently import the active NF- $\mathrm{kB}$ into the nucleus [26]. More and more studies have indicated that miRNAs exert vital roles in myocardial IR injury by regulating NF- $\mathrm{kB}$ pathway $[9,18,27]$. Li et al. [9] have confirmed that ron-miR-340-5p suppresses hypoxia/reoxygenation-induced apoptosis and oxidative stress in myocardial $\mathrm{H} 9 \mathrm{C} 2$ cells via regulating Act1/NF- 
$\mathrm{\kappa B}$ signaling. Liu et al. [27] have reported that the inhibition of mmu-miR-27a induces high thoracic epidural block to protect mice against myocardial IR injury via activating NF- $\mathrm{KB}$ pathway. In this study, overexpression and silencing of rno-miR-30c-5p significantly activated and blocked NF- $\kappa B$ pathway in IR myocardial cells. We speculate that rno-miR-30c-5p may promote the inflammation and apoptosis of myocardial cells in rats with myocardial IR injury through activating NF- $\kappa$ B pathway.

SIRT1 is a member of the sirtuin family that involved in the regulation of cell proliferation, apoptosis and autophagy [28, 29]. Emerging researches have indicated that SIRT1 is a potential therapeutic target for myocardial IR injury [30]. Yu et al. [31] have indicated that melatonin ameliorates IR-induced oxidative stress and endoplasmic reticulum stress via activating SIRT1 signaling in type 2 diabetic rats. Wang et al. [32] have proved that post-ischemic treatme

nt with lumbrokinase attenuates myocardial IR injury through the activation of Sirt1 signaling. Lin et al. [33] have demonstrated that the activation of SIRT1/Nrf2 signaling induced by Rutin contributes to the reduced oxidative stress and apoptosis of cardiomyocytes in rats with myocardial IR injury. Notably, a recent study showed that rno-miR-34a increases the apoptosis and infarct size and decreases left ventricular function through negatively regulating SIRT1 in rats with myocardial IR injury [34]. In this study, SIRT1 was identified as a target gene of rno-miR-30c-5p. We speculate that the upregulation of SIRT1 may contribute to the promoting effect of rno-miR-30c-5p on myocardial IR injury. This speculation was further illustrated by that silencing of SIRT1 reversed the effects of rno-miR-30c-5p inhibitor on the apoptosis and NF-kB pathway in IR myocardial cells. Evidence has shown that SIRT1 inhibits the transcription of NF- $\mathrm{kB}$ through the deacetylation of NF- $\mathrm{kB}$ $[35,36]$. The up-regulation of SIRT1 may relieve myocardial IR injury through blocking NF-kB signaling.

This study has some limitations. First, the regulatory role of rno-miR-30c-5p on myocardial IR injury is limited at the cellular level. The therapeutic effect of rno-miR-30c-5p silencing on rats with myocardial IR injury remains to be studied. Second, only rno-miR-30c-5p was studied. More miRNAs involving myocardial IR injury still need to be discovered based on microarray or RNA-seq methodologies. Third, only one target of rnomiR-30c-5p was selected. The discovery of more targets of rno-miR-30c-5p based on omics measurements is needed.

\section{Conclusions}

In conclusion, rno-miR-30c-5p was up-regulated in rats with myocardial IR injury. Rno-miR-30c-5p enhanced the inflammation, promoted the apoptosis, and activated
NF-kB pathway in IR myocardial cells through targeting SIRT1. Rno-miR-30c-5p may promote the myocardial IR injury in rats through activating NF- $\mathrm{kB}$ pathway and down-regulating SIRT1. Our research discovers a novel regulatory mechanism of rno-miR-30c-5p in myocardial IR injury and points out a novel therapeutic target.

\section{Supplementary information}

Supplementary information accompanies this paper at https://doi.org/10. 1186/s12872-020-01520-2.

Additional File 1. Table S1. miR-30-5p-all predicted transcripts

Additional File 2. Table S2. Predicted details of SIRT1R4

\section{Abbreviations}

LAD: Left anterior descending; mIRNAs: Micrornas; IR: Ischemia reperfusion

Acknowledgements

Not applicable.

\section{Authors' contributions}

JFC and SFX: conception, design and analysis of data, performed the data analyses and wrote the manuscript; JFC and MMZ: contributed to the conception of the study and revised the manuscript; JLW: contributed to the conception of the study; SYZ: contributed significantly to analysis and manuscript preparation and revised the manuscript; All authors have read and approved the manuscript.

\section{Funding}

Not applicable.

\section{Availability of data and materials}

The datasets used and/or analysed during the current study are available from the corresponding author on reasonable request. The genes analyzed in the present study are available at https://www.ncbi.nlm.nih.gov/search/ with Gene ID: 100314012 (microRNA-30c-5p, ENSMUSG00000065567; http:// asia.ensembl.org/Mus_musculus/Gene/Summary?db=core; $g=$

ENSMUSG00000065567;r=1:23291701-23291784;t=ENSMUST00000083633), and Gene ID: 309757 (Sirtuin 1, ENSMUSG00000020063; http://asia.ensembl. org/Mus_musculus/Gene/Summary?db=core;g=ENSMUSG00000020063;r= 10:63319005-63381704)

Ethics approval and consent to participate

This study was conducted after obtaining Luoyang Central Hospital Affiliated to Zhengzhou University's ethical committee approval.

Consent for publication

Not applicable.

\section{Competing interests}

The authors declare that they have no competing interests.

\section{Author details}

${ }^{1}$ Department of Cardiology, Luoyang Central Hospital Affiliated to Zhengzhou University, No. 288, Zhongzhou Middle Road, Luoyang City 471000, Henan Province, China. ${ }^{2}$ Department of Orthopedics, Luoyang Central Hospital Affiliated to Zhengzhou University, No. 288, Zhongzhou Middle Road, Luoyang City 471000, Henan Province, China.

Received: 20 September 2019 Accepted: 10 May 2020 Published online: 20 May 2020

\section{References}

1. Wong ND. Epidemiological studies of CHD and the evolution of preventive cardiology. Nat Rev Cardiol. 2014;11:276-89.

2. Ovize M, Kloner RA, Hale SL, Przyklenk K. Coronary cyclic flow variations "precondition" ischemic myocardium. Circulation. 1992;85:779-89. 
3. Cohn JN, Ferrari R, Sharpe N. Cardiac remodeling--concepts and clinical implications: a consensus paper from an international forum on cardiac remodeling. Behalf of an international forum on cardiac remodeling. J Am Coll Cardiol. 2000;35:569-82.

4. Perrino C, Barabási A-L, Condorelli G, Davidson SM, De Windt L, Dimmeler S, et al. Epigenomic and transcriptomic approaches in the post-genomic era: path to novel targets for diagnosis and therapy of the ischaemic heart? Position paper of the European Society of Cardiology Working Group on cellular biology of the heart. Cardiovasc Res. 2017;113:725-36.

5. Guo H, Ingolia NT, Weissman JS, Bartel DP. Mammalian microRNAs predominantly act to decrease target mRNA levels. Nature. 2010;466:835-40.

6. Ebert MS, Sharp PA. Roles for microRNAs in conferring robustness to biological processes. Cell. 2012;149:515-24.

7. Varga ZV, Zvara Á, Faragó N, Kocsis GF, Pipicz M, Gáspár R, et al. MicroRNAs associated with ischemia-reperfusion injury and cardioprotection by ischemic pre-and postconditioning: protectomiRs. Am J Phys Heart Circ Phys. 2014;307:H216-H27.

8. Schulz R, Ágg B, Ferdinandy P. Survival pathways in cardiac conditioning: individual data vs. meta-analyses. What do we learn? Basic Res Cardiol. 2018;113:4.

9. Li D, Zhou J, Yang B, Yu Y. microRNA-340-5p inhibits hypoxia/ reoxygenation-induced apoptosis and oxidative stress in cardiomyocytes by regulating the Act1/NF-kappaB pathway. J Cell Biochem. 2019;120:14618-27.

10. Yuan L, Fan L, Li Q, Cui W, Wang X, Zhang Z. Inhibition of miR-181b-5p protects cardiomyocytes against ischemia/reperfusion injury by targeting AKT3 and PI3KR3. J Cell Biochem. 2019;120(12):19647.

11. Zhao-Qi H, Wei X, Jin-Lei W, Xiong L, Xi-Ming C. MicroRNA-374a protects against myocardial ischemia-reperfusion injury in mice by targeting the MAPK6 pathway. Life Sci. 2019;232:116619.

12. Song $C L$, Liu B, Wang JP, Zhang BL, Zhang JC, Zhao LY, et al. Anti-apoptotic effect of microRNA-30b in early phase of rat myocardial ischemiareperfusion injury model. J Cell Biochem. 2015;116:2610-9.

13. Zou YF, Wen D, Zhao Q, Shen PY, Shi H, Chen YX, et al. Urinary MicroRNA30c-5p and MicroRNA-192-5p as potential biomarkers of ischemiareperfusion-induced kidney injury. Exp Biol Med (Maywood). 2017:242:657-67.

14. Li L, Jiang HK, Li YP, Guo YP. Hydrogen sulfide protects spinal cord and induces autophagy via miR-30c in a rat model of spinal cord ischemiareperfusion injury. J Biomed Sci. 2015;22:50.

15. Gao Y, Jiang W, Dong C, Li C, Fu X, Min L, et al. Anti-inflammatory effects of sophocarpine in LPS-induced RAW 264.7 cells via NF-kappaB and MAPKs signaling pathways. Toxicol In Vitro. 2012;26:1-6.

16. van Delft MA, Huitema LF, Tas SW. The contribution of NF-kappaB signalling to immune regulation and tolerance. Eur J Clin Investig. 2015;45:529-39.

17. Chandrasekar B, Smith JB, Freeman GL. Ischemia-reperfusion of rat myocardium activates nuclear factor-KappaB and induces neutrophil infiltration via lipopolysaccharide-induced CXC chemokine. Circulation. 2001; 103:2296-302

18. Wang X, Ha T, Liu L, Zou J, Zhang X, Kalbfleisch J, et al. Increased expression of microRNA-146a decreases myocardial ischaemia/reperfusion injury. Cardiovasc Res. 2013;97:432-42

19. Yu Y, Zhang M, Hu Y, Zhao Y, Teng F, Lv X, et al. Increased bioavailable Berberine protects against myocardial ischemia reperfusion injury through attenuation of NFkappaB and JNK signaling pathways. Int Heart J. 2018;59: 1378-88.

20. Hausenloy DJ, Yellon DM. Myocardial ischemia-reperfusion injury: a neglected therapeutic target. J Clin Invest. 2013;123:92-100.

21. Wang Z, Yu J, Wu J, Qi F, Wang H, Xu Z. Scutellarin protects cardiomyocyte ischemia-reperfusion injury by reducing apoptosis and oxidative stress. Life Sci. 2016;157:200-7.

22. Mishra PK, Tyagi N, Kumar M, Tyagi SC. MicroRNAs as a therapeutic target for cardiovascular diseases. J Cell Mol Med. 2009;13:778-89.

23. Fan $Z X$, Yang J. The role of microRNAs in regulating myocardial ischemia reperfusion injury. Saudi Med J. 2015;36:787-93.

24. Chen Z, Su X, Shen Y, Jin Y, Luo T, Kim IM, et al. MiR322 mediates cardioprotection against ischemia/reperfusion injury via FBXW7/notch pathway. J Mol Cell Cardiol. 2019;133:67-74.

25. LE, Jiang H, Lu Z. MicroRNA-144 attenuates cardiac ischemia/reperfusion injury by targeting FOXO1. Exp Ther Med. 2019;17:2152-60.

26. Huang W, Cui X, Chen J, Feng Y, Song E, Li J, et al. Long non-coding RNA NKILA inhibits migration and invasion of tongue squamous cell carcinoma cells via suppressing epithelial-mesenchymal transition. Oncotarget. 2016;7: 62520-32.

27. Liu JY, Shang J, Mu XD, Gao ZY. Protective effect of down-regulated microRNA-27a mediating high thoracic epidural block on myocardial ischemia-reperfusion injury in mice through regulating ABCA1 and NFkappaB signaling pathway. Biomed Pharmacother. 2019;112:108606.

28. Karbasforooshan $\mathrm{H}$, Roohbakhsh A, Karimi G. SIRT1 and microRNAs: the role in breast, lung and prostate cancers. Exp Cell Res. 2018;367:1-6.

29. Poulose N, Raju R. Sirtuin regulation in aging and injury. Biochim Biophys Acta. 1852;2015:2442-55.

30. Pantazi E, Zaouali MA, Bejaoui M, Folch-Puy E, Ben Abdennebi H, RoselloCatafau J. Role of sirtuins in ischemia-reperfusion injury. World J Gastroenterol. 2013;19:7594-602.

31. Yu L, Liang H, Dong X, Zhao G, Jin Z, Zhai M, et al. Reduced SIRT1 signaling exacerbates myocardial ischemia reperfusion injury in type 2 diabetic rats and the protective effect of melatonin. J Pineal Res. 2015;59:376-90.

32. Wang YH, Shun-An L, Chao-Hsin H, Hsing-Hui S, Yi-Hung C, T CJ, et al. Sirt1 Activation by Post-ischemic Treatment With Lumbrokinase Protects Against Myocardial Ischemia-Reperfusion Injury. Front Pharmacol. 2018;9:636.

33. Qiao L, Xiu-Ying C, Ji Z, Yong-Liang Y, Wen Z, Bo W. Upregulation of SIRT1 contributes to the cardioprotective effect of Rutin against myocardial ischemia-reperfusion injury in rats. J Functional Foods. 2018:46:227-36.

34. Fu BC, Lang J-L, Zhang D-Y, Sun L, Chen W, Liu W, et al. Suppression of miR-34a expression in the myocardium protects against ischemiareperfusion injury via SIRT1 protective pathway. Stem Cells \& Development. scd; 2017. p. 0062

35. Haigis MC, Sinclair DA. Mammalian sirtuins: biological insights and disease relevance. Annu Rev Pathol. 2010;5:253-95.

36. Yeung F, Hoberg JE, Ramsey CS, Keller MD, Jones DR, Frye RA, et al. Modulation of NF-kappaB-dependent transcription and cell survival by the SIRT1 deacetylase. EMBO J. 2004;23:2369-80.

\section{Publisher's Note}

Springer Nature remains neutral with regard to jurisdictional claims in published maps and institutional affiliations.

\section{Ready to submit your research? Choose BMC and benefit from:}

- fast, convenient online submission

- thorough peer review by experienced researchers in your field

- rapid publication on acceptance

- support for research data, including large and complex data types

- gold Open Access which fosters wider collaboration and increased citations

- maximum visibility for your research: over $100 \mathrm{M}$ website views per year

At BMC, research is always in progress.

Learn more biomedcentral.com/submissions 